\title{
Roles of Education and IQ in Cognitive Reserve in Parkinson's Disease-Mild Cognitive Impairment
}
M.J. Armstrong ${ }^{a, b}$
G. Naglie $\mathrm{c-e}$
S. Duff-Canning ${ }^{f}$
C. Meaney ${ }^{9}$
D. Gill ${ }^{\mathrm{h}}$ P.J. Eslinger ${ }^{\mathrm{h}}$
C. Zadikoff ${ }^{i}$
M. Mapstone ${ }^{j}$
K.L. Chou ${ }^{k}$
C. Persad' I. Litvan ${ }^{m}$
B.T. Mast ${ }^{n}$
S. Fox ${ }^{a, b}$
D.F. Tang-Wai ${ }^{b, o}$
C. Marras ${ }^{a, b}$

aToronto Western Hospital Movement Disorders Centre and the Edmond J. Safra Program in Parkinson's Disease, 'b Department of Medicine (Neurology), University of Toronto, 'Department of Medicine and the Rotman Research Institute, Baycrest Geriatric Health Care Centre, ${ }^{\mathrm{d}}$ Department of Medicine and ${ }^{\mathrm{e}}$ Institute of Health Policy, Management and Evaluation, University of Toronto, ${ }^{f}$ Department of Neuropsychology, Toronto Western Hospital, and ${ }^{9}$ Department of Family and Community Medicine, University of Toronto,

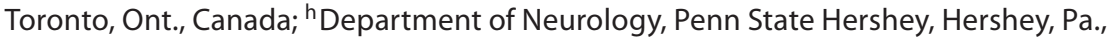
'Department of Neurology, Northwestern University, Evanston, III., jDepartment of Neurology, University of Rochester, Rochester, N.Y., Departments of kNeurology and 'Psychiatry, Ann Arbor, Mich., 'm Department of Neurology, University of California, San Diego, Calif., and ${ }^{n}$ Psychological and Brain Sciences, University of Louisville, Louisville, Ky., USA; ' University Health Network Memory Clinic, Division of Neurology, University of Toronto, Toronto, Ont., Canada

\section{Key Words}

Parkinson's disease $\cdot$ Mild cognitive impairment $\cdot$ Education $\cdot I Q \cdot$ Cognitive reserve

\begin{abstract}
Background/Aims: The role of cognitive reserve in Parkinson's disease (PD)-mild cognitive impairment $(\mathrm{MCl})$ is incompletely understood. Methods: The relationships between PD-MCl, years of education, and estimated premorbid IQ were examined in 119 consecutive non-demented PD patients using logistic regression models. Results: Higher education and IQ were associated with reduced odds of PD-MCl in univariate analysis. In multivariable analysis, a higher IQ was associated with a significantly decreased odds of PD-MCl, but education was not. Conclusion: The association of higher IQ and decreased odds of PD-MCl supports a role for cognitive reserve in PD, but further studies are needed to clarify the interaction of IQ and education and the impact of other contributors such as employment and hobbies.

Copyright $\odot 2012$ S. Karger AG, Basel
\end{abstract}




\section{Introduction}

Cognitive reserve is the concept that individual differences in neural pathways and cognitive processes may affect a person's ability to compensate for brain pathology. Certain factors and life experiences such as IQ, education, employment, and leisure activities are proposed to increase cognitive reserve and are frequently used as indicators or markers of cognitive reserve. While much cognitive reserve research has been in Alzheimer's disease (AD), the concept is also applicable to other neurological diseases [1]. Studies describing the effect of education on Parkinson's disease (PD)-mild cognitive impairment (MCI) have produced mixed results [2-5]. We aimed to investigate the relationship between a diagnosis of PD-MCI and (1) education level (the primary variable of interest) and (2) IQ (the secondary variable of interest) as theorized indicators of cognitive reserve in a well-characterized cohort of nondemented PD patients.

\section{Patients and Methods}

\section{Subjects}

Consecutive English-speaking non-demented PD patients were enrolled at six North American movement disorders centers as part of an ongoing prospective longitudinal study of PD-MCI screening measures. Recruitment started in December 2008 and continued through June 2011; annual follow-up visits are ongoing. Inclusion criteria were a diagnosis of PD according to the United Kingdom PD Society Brain Bank Criteria, age $\geq 60$ years, English as first language, grade 8 or higher education, and a standard score of at least 80 on the Wechsler Test of Adult Reading (WTAR), a standardized estimate of premorbid IQ. Language and minimum education/IQ inclusion criteria were prerequisites chosen for accurate interpretation and comparison of neuropsychological testing results.

Exclusion criteria included evidence of impairments in activities of daily living or instrumental activities of daily living related to cognition on a modified Disability Assessment for Dementia (DAD), medication changes over the past month, planned medication changes over the next 3 weeks, color blindness, a score of $\geq 5$ on the 15-item Geriatric Depression Scale, currently unstable psychiatric disorder, history of prior stroke, brain tumor, hospitalization for head injury, or active central nervous system illness (e.g. multiple sclerosis), prior or planned neurosurgical intervention (e.g. deep brain stimulation), enrollment in another interventional or observational clinical trial using neuropsychological testing, neuropsychological testing in the past 12 months, and lack of consent from either the subject or an identified close contact. Enrolled subjects received a clinical evaluation followed 1-3 weeks later by formal neuropsychological testing performed blinded to clinical results.

Each participating institution received local research ethics board approval prior to study enrollment. Written informed consent was obtained from all study subjects and participating close contacts (defined as contact at least twice weekly) prior to formal screening and study visits.

\section{Clinical Assessment}

The clinical evaluation included the Montreal Cognitive Assessment (MoCA), MiniMental State Examination (MMSE), Neurobehavioral Signs and Symptoms Abbreviated Inventory (NBI) (@ Professional Resources and Technologies, Westtown, Pa., USA), and Movement Disorders Society Unified Parkinson's Disease Rating Scale (MDS-UPDRS). Historical information was provided by the subject. The number of years of education (YOE) was assigned by patient report of the highest grade attended (up to grade 12 or 13 for secondary 
school) plus any additional years for post-secondary training. Post-secondary degrees were assigned YOE based on typical completion time after secondary school. Bachelor's degrees were coded as 16 YOE, master's degrees as $18 \mathrm{YOE}$, and doctorates as $20 \mathrm{YOE}$.

A consenting close contact completed the NBI and a modified version of the DAD in reference to the subject. The modified DAD added a question regarding whether any reported impairment was related to cognitive difficulties or the physical impairments of PD.

\section{Neuropsychological Testing}

Neuropsychological testing was performed 1-3 weeks following the clinical evaluation to minimize the risks of status changes in the intervening time and potential interference from similar tasks on clinical and neuropsychological cognitive evaluations. The WTAR was used as an estimate of premorbid IQ. The neuropsychological battery included the Wechsler Memory Scale-III (WMS-III) Digit Span and Letter-Number Sequencing Test [6], the Trail Making Test [7], Delis-Kaplan Executive Function System (D-KEFS) Color-Word Interference Test and verbal fluency subtest [8], Benton Judgment of Line Orientation (JLO) [9], Rey Complex Figure Test and Recognition Trial [10], California Verbal Learning Test-II (CVLTII) [11], modified conditional associative learning test [12], Visual Verbal Test (VVT) abbreviated 10-item version [13], Frontal Systems Behavior Scale [14], and the 30-item Boston Naming Test (odd version) [15].

\section{Diagnosis of PD-MCI}

Diagnosis of PD-MCI was made by consensus conference based on modified Petersen criteria [2] and required (1) a cognitive complaint from the subject or close contact on the NBI, (2) no functional impairment related to cognition (as assessed by the modified DAD), and (3) performance at least $1.5 \mathrm{SD}$ below the age-adjusted mean on at least one of eight core neuropsychological variables designated a priori. Core variables were WMS-III Digit Span longest span forward (attention), D-KEFS time to complete Condition 1 from the ColorWord Interference Test (processing speed), CVLT-II long delay free recall score (learning and memory), D-KEFS total score for category fluency condition from the verbal fluency subtest (language), JLO total correct (visuospatial), WMS-III total score from the Letter-Number Sequencing Test (executive: working memory), VVT total number of shifts (executive: cognitive flexibility), and D-KEFS time to complete Condition 3 from the Color-Word Interference Test (executive: inhibition). CVLT-II and JLO scores were adjusted for gender in addition to age, and VVT norms were from a control group with similar age and education to the study population.

\section{Data Analysis}

Baseline demographic characteristics were summarized as medians and interquartile ranges (IQR) or percentages as appropriate. Spearman's correlation coefficients were calculated for the relationships between each demographic variable and YOE, the primary variable of interest.

Logistic regression was performed to estimate the effect of YOE (the primary variable of interest) and IQ (the second variable of interest) on a diagnosis of PD-MCI in both univariate and multivariable models. Variables considered a priori for the multivariable model were YOE, IQ, age, age at PD diagnosis, PD duration, gender, PD severity as assessed by the MDSUPDRS total and motor scores, postural instability and gait disorder (PIGD) subtype [calculated as a 5-point score of the sum of walking difficulty and freezing by history and gait, freezing, and postural stability by examination, where each was dichotomized as 0 (normal) or 1 (abnormal >0)], levodopa-equivalent units, and the presence of comorbidities occurring in over $10 \%$ of subjects: hypertension, hyperlipidemia, coronary artery disease, or any one of 
Dement Geriatr Cogn Disord Extra 2012;2:343-352

DOI: 10.1159/000341782

Published online: August 22, 2012

() 2012 S. Karger AG, Base

www.karger.com/dee

Armstrong et al.: Roles of Education and IQ in Cognitive Reserve in Parkinson's

Disease-Mild Cognitive Impairment

these three (a combined cardiovascular risk variable). An interaction between YOE and IQ was hypothesized, and a product term including these two variables was tested for significance. The number of independent variables included in the final model was reduced based on the available sample size $[16,17]$. Variable reduction was performed by the investigation of the multicollinearity and application of the Harrell variable reduction method using YOE as the independent variable of interest [18]. Odds ratios (ORs) and 95\% CIs were calculated from the final logistic regression model.

For the secondary analysis, subjects were categorized into low- and high-education groups according to whether they attained post-secondary schooling or not. This division is common in the literature and is a relevant cutoff for North American populations. The loweducation group included subjects whose highest education was up to high school graduation (grade 12 or 13 depending on location) and the high-education group included subjects with at least 1 year of post-secondary schooling. The univariate and multivariable logistic regression models developed above were re-derived replacing YOE with high versus low education.

Secondary outcomes were PD-MCI defined without the criterion of a cognitive complaint and performance on individual neuropsychological measures. For the association between education and PD-MCI without the requirement of a cognitive complaint (i.e. cognitive impairment as determined by scores $\geq 1.5 \mathrm{SD}$ below the mean on at least one core neuropsychological measure without evidence of impairment in function related to cognition), the univariate and multivariable regression models were re-derived for IQ, YOE, and dichotomized education level. For the association of the core neuropsychological measures with education, median and IQR scores for the low- and high-education groups were compared using Wilcoxon rank sum statistics. Spearman's correlation coefficient was calculated for each core measure and YOE.

Complete data were available for every variable of interest except for PIGD, for which the analysis was performed without the single missing subject. Significance was assigned $\mathrm{p}<$ 0.05. All analyses were performed using SAS statistical software version 9.2 (SAS Institute).

\section{Results}

\section{Baseline Characteristics}

In total, 157 consecutive non-demented PD patients consented to participate in the study. Thirty (19\%) did not meet the inclusion criteria, and an additional 8 (3\%) were excluded after their first clinical assessment (fig. 1). At the time of analysis, 119 subjects had complete year-1 assessments. YOE was significantly correlated with age, gender, age at PD diagnosis, and IQ, though all Spearman's correlation coefficients had an absolute value of 0.35 or less except for the coefficient for IQ (table 1). Uncorrected MMSE and MoCA scores had low but significant correlations with YOE (table 1), but the MoCA correlation was no longer significant when the extra point was given for $\leq 12$ years of formal education as recommended in the scoring instructions [19].

Univariate Modeling of PD-MCI

A total of 61 of 119 subjects (51\%) were diagnosed with PD-MCI, and 77 subjects (65\%) were categorized as PD-MCI if no cognitive complaint was required. Univariate ORs for the associations of education and IQ with both diagnoses are presented in table 2 .

\section{Multivariable Modeling of PD-MCI}

The final multivariable model for PD-MCI included YOE, IQ, and the clinically relevant variables age, gender, and total MDS-UDPRS score. During Harrell variable reduction [18], 
Armstrong et al.: Roles of Education and IQ in Cognitive Reserve in Parkinson's

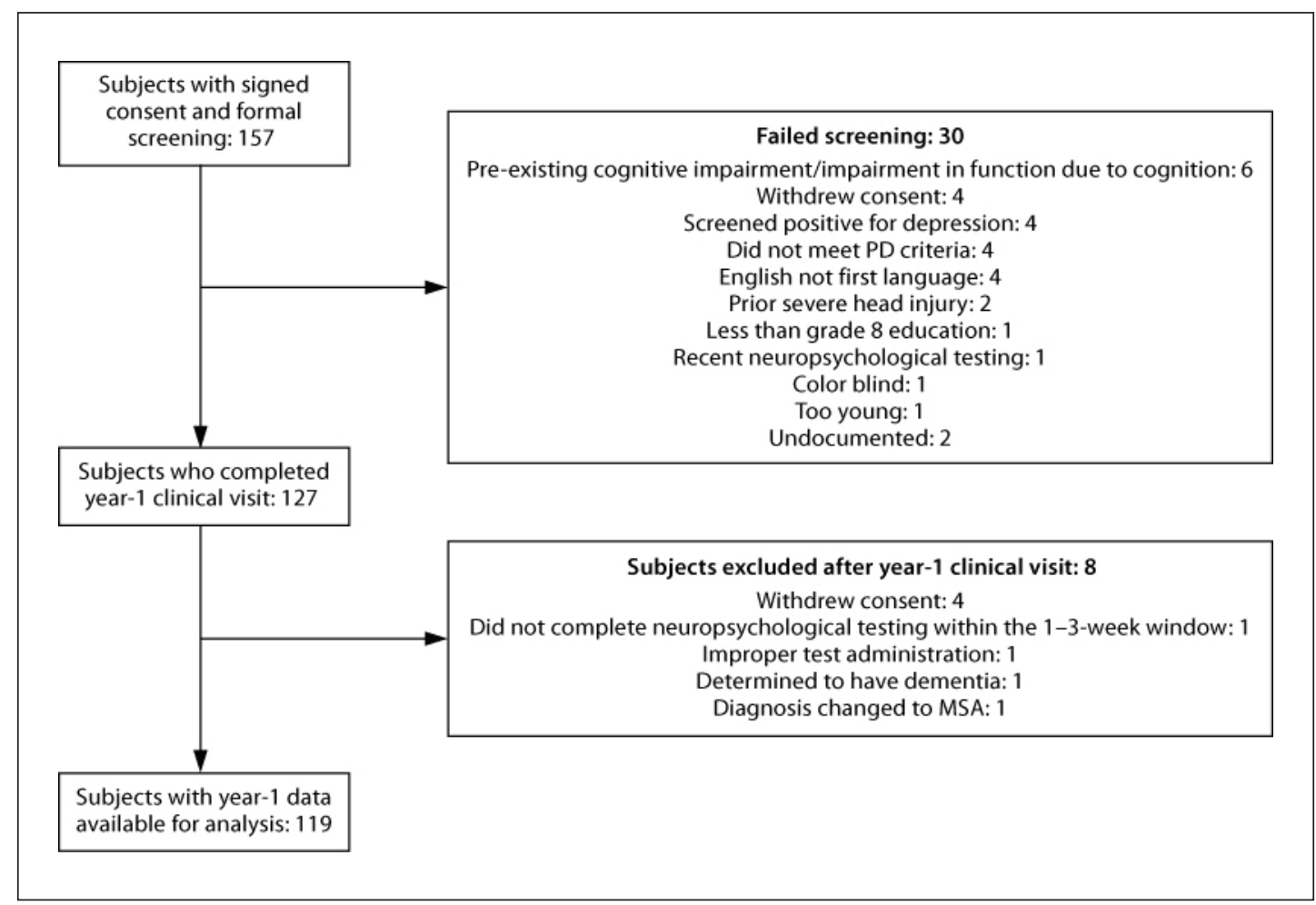

Fig. 1. Study enrollment flow chart. MSA = Multiple System Atrophy

Table 1. Baseline characteristics

\begin{tabular}{lccc}
\hline Variable & $\begin{array}{l}\text { Total } \\
\text { population }\end{array}$ & $\begin{array}{c}\text { Correlation } \\
\text { with YOE }\end{array}$ & $\begin{array}{c}\text { Two-sided } \\
\text { p value }\end{array}$ \\
\hline Patients & 119 & - & - \\
Age, years & $71(67-75)$ & -0.23 & 0.01 \\
Male gender & $69 \%$ & 0.35 & 0.001 \\
Age at PD diagnosis, years & $66(62-71)$ & -0.19 & 0.04 \\
Years since diagnosis & $4(2-8)$ & 0.03 & 0.74 \\
YOE & $16(15-18)$ & 1.0 & - \\
Premorbid IQ & $116(109-122)$ & 0.64 & 0.0001 \\
Total MDS-UPDRS score & $42(32-54)$ & 0.11 & 0.24 \\
Motor MDS-UPDRS score & $26(20-34)$ & -0.14 & 0.11 \\
PIGD score (maximum 5) & $2(1-3)$ & -0.15 & 0.85 \\
Total LEU, mg & $450.0(300.0-787.5)$ & -0.02 & 0.64 \\
Hypertension & $34 \%$ & -0.04 & 0.46 \\
Hyperlipidemia & $28 \%$ & 0.068 & 0.49 \\
Coronary artery disease & $18 \%$ & -0.064 & 0.48 \\
Any of the 3 cardiovascular risk factors & $49 \%$ & -0.065 & 0.02 \\
MoCA score (without EL bonus point) & $26(23-27)$ & 0.22 & 0.07 \\
MoCA score (with EL bonus point) & $26(23-27)$ & 0.17 & 0.05 \\
MMSE score & $29(27-30)$ & 0.18 & \\
\hline
\end{tabular}

Values are medians (IQR, 25th and 75th percentiles) or percentages.

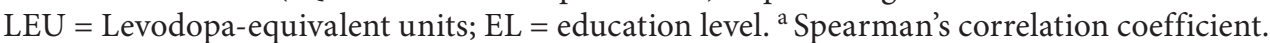

${ }^{\mathrm{b}} 118$ subjects. 
Table 2. Odds of PD-MCI (diagnosed with and without the requirement for a cognitive complaint) based on education and IQ

\begin{tabular}{|c|c|c|c|c|}
\hline \multirow[t]{2}{*}{ Variable } & \multicolumn{2}{|c|}{$\begin{array}{l}\text { Odds ratio of PD-MCI with a cognitive complaint } \\
(95 \% \mathrm{CI}) \text {; } \mathrm{p} \text { value }\end{array}$} & \multicolumn{2}{|c|}{$\begin{array}{l}\text { Odds ratio of PD-MCI without a cognitive complaint } \\
\text { (95\% CI); p value }\end{array}$} \\
\hline & univariable analysis & multivariable analysis ${ }^{\mathrm{a}}$ & univariable analysis & multivariable analysis $^{\mathrm{a}}$ \\
\hline YOE (1-year increment) & 0.83 (0.71-0.97); 0.02 & $1.06(0.85-1.31) ; 0.63$ & 0.84 (0.71-0.99); 0.03 & $0.94(0.75-1.17) ; 0.57$ \\
\hline High education $^{\mathrm{b}}$ & $0.19(0.06-0.61) ; 0.005$ & $0.46(0.12-1.78) ; 0.26$ & $0.15(0.03-0.69) ; 0.01$ & $0.24(0.04-1.30) ; 0.10$ \\
\hline \multirow[t]{4}{*}{ IQ (5-point increment) } & $0.61(0.47-0.79) ; 0.0002$ & YOE model: & $0.68(0.52-0.88) ; 0.004$ & YOE model: \\
\hline & & 0.60 (0.44-0.83); 0.002 & & $0.67(0.47-0.94) ; 0.02$ \\
\hline & & High-education model: & & High-education model: \\
\hline & & $0.67(0.50-0.89) ; 0.006$ & & $0.70(0.51-0.96) ; 0.03$ \\
\hline
\end{tabular}

${ }^{\mathrm{a}}$ Variables in the model included education level (YOE or high education), IQ, age, gender, and total MDS-UPDRS score. ${ }^{\mathrm{b}}$ High education is defined as 1 year of post-secondary education or more.

Table 3. Association between core neuropsychological measures and level of education

\begin{tabular}{|c|c|c|c|c|c|}
\hline Domain & Test & $\begin{array}{l}\text { Low education } \\
(\mathrm{n}=21)\end{array}$ & $\begin{array}{l}\text { High education } \\
(\mathrm{n}=98)\end{array}$ & $\begin{array}{l}\text { Two- } \\
\text { sided } \\
\mathrm{p} \\
\text { value }\end{array}$ & $\begin{array}{l}\text { Correlation } \\
\text { with } \mathrm{YOE}^{\mathrm{a}}\end{array}$ \\
\hline Attention & $\begin{array}{l}\text { WMS-III Digit Span - } \\
\text { longest span forward }\end{array}$ & $0.51(-0.05$ to 0.88$)$ & $0.62(-0.10$ to 1.21$)$ & 0.68 & $0.11(\mathrm{p}=0.23)$ \\
\hline Processing speed & $\begin{array}{l}\text { D-KEFS Color-Word Interference Test - } \\
\text { time to complete Condition } 1 \text { (color } \\
\text { naming) }\end{array}$ & $0.33(-0.33$ to 0.67$)$ & $0.0(-0.67$ to 0.67$)$ & 0.32 & $-0.13(\mathrm{p}=0.17)$ \\
\hline Learning and memory & CVLT-II - long delay free recall & $0.0(-1.0$ to 1.0$)$ & $0.0(-0.5$ to 1.0$)$ & 0.55 & $0.14(\mathrm{p}=0.14)$ \\
\hline Language & $\begin{array}{l}\text { D-KEFS verbal fluency test - } \\
\text { category fluency }\end{array}$ & $0.0(-0.67$ to 0.67$)$ & $0.33(-0.33$ to 0.99$)$ & 0.14 & $0.033(\mathrm{p}=0.72)$ \\
\hline Visuospatial & Benton JLO & $-0.70(-1.97$ to -0.07$)$ & $-0.27(-1.12$ to 1.00$)$ & 0.06 & $0.10(\mathrm{p}=0.27)$ \\
\hline Executive (working memory) & WMS-III Letter-Number Sequencing & $0.33(-0.67$ to 0.99$)$ & $0.33(0.00$ to 0.99$)$ & 0.45 & $0.060(\mathrm{p}=0.52)$ \\
\hline Executive (cognitive flexibility) & VVT - total number of shifts & $-1.99(-4.88$ to -1.99$)$ & $-1.03(-1.99$ to -0.07$)$ & 0.001 & $0.30(\mathrm{p}=0.001)$ \\
\hline Executive (inhibition) & $\begin{array}{l}\text { D-KEFS Color-Word Interference Test - } \\
\text { time to complete Condition } 3 \text { (inhibition) }\end{array}$ & $0.33(-0.67$ to 0.99$)$ & $0.33(-0.67$ to 0.99$)$ & 0.59 & $-0.026(\mathrm{p}=0.78)$ \\
\hline
\end{tabular}

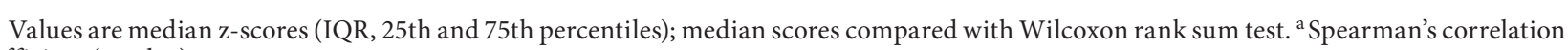
coefficient (p value).

IQ was the only variable that significantly modified the relationship between YOE and MCI. The interaction term of YOE and IQ was not significant and was not included in the final model.

In the final multivariable model, IQ significantly decreased the odds of PD-MCI in both the YOE and high-education multivariable models (table 2). The association between higher education and decreased odds of PD-MCI was no longer significant. IQ and YOE were highly correlated (Spearman's correlation coefficient 0.64; $\mathrm{p}<0.0001]$ ). When IQ was removed from the final model to test if YOE was non-significant due to the inclusion of both YOE and IQ, the smaller (nested) model had a higher Akaike Information Criteria [20] value, suggesting that the full model including IQ and YOE had better relative goodness of fit than the model excluding IQ. Thus, the 5-variable model was maintained.

When MCI was defined without requiring a cognitive complaint, IQ was again the only significant predictor in the 5-variable model. The ORs for PD-MCI without the requirement of a cognitive complaint are presented in table 2. A higher IQ again reduced the odds of PD- 
Armstrong et al.: Roles of Education and IQ in Cognitive Reserve in Parkinson's

Disease-Mild Cognitive Impairment

MCI, while the benefit of higher education seen in univariate analysis was lost in the multivariable models.

\section{Neuropsychological Measures and Education Level}

The relationship between the core neuropsychological variables and education, the primary variable of interest, is shown in table 3 . Patients in the low-education group scored significantly lower on the core test for executive cognitive flexibility, which was also the only test that correlated significantly with YOE. There was a trend for lower visuospatial scores in the low-education group (table 3).

\section{Discussion}

A higher level of education was associated with decreased odds of PD-MCI in univariate analyses, but not in multivariable logistic regression models controlling for IQ, age, gender, and total MDS-UPDRS score. In these models, the point ORs suggested that higher education may decrease the odds of PD-MCI, but the results were not statistically significant. Estimated premorbid IQ, however, was a strong predictor of PD-MCI in all models, a relationship reported only once previously [5]. This is consistent with recent theories of cognitive reserve, where some AD studies have shown that IQ is a more powerful predictor of cognitive reserve than YOE [21], but education and other experiences impart additional benefit on top of IQ alone [1].

Prior studies with education levels similar to those in our study are mixed regarding whether education is associated with the development of PD-MCI. The lack of consensus regarding PD-MCI criteria, methods to operationalize the commonly used Petersen criteria, and selection of neuropsychological domains, tests, and cutoffs for diagnosing PD-MCI currently limits the comparability of studies. Keeping these limitations in mind, some studies found no association between YOE and PD-MCI [2-4], while another found that PD-MCI patients had significantly lower YOE [5]. Importantly, the latter was the only study that also estimated premorbid IQ. While ORs were not reported, IQ and YOE were both significantly associated with PD-MCI in univariate analysis [5]. Multivariable analysis with these variables was not performed. Thus, our study is the first, to our knowledge, to investigate the association between education and PD-MCI taking premorbid IQ into account.

Our results and those of studies enrolling similar patients are applicable only to welleducated populations. However, they should be generalizable within the United States and Canada, where $88-89 \%$ of adults have completed at least an upper secondary (high school) education and $41-50 \%$ have completed a post-secondary degree [22]. The literature in populations with less education is also mixed but generally suggestive of an association between education level and cognitive performance in at least some domains [23-26]. Most standardized cognitive tests require some degree of literacy, limiting the ability to apply the measures to the lowest end of the educational spectrum. Additionally, most standardized cognitive tests, including the ones used in this study, do not have education-stratified norms for total and/or subdomain scores [7]. Available norms are likely relevant for our population as norms for most of the large cognitive batteries (e.g. WMS-III) were developed using stratified samples based on the US census [7] and education levels in the United States are generally similar to those in our study [22].

One challenge in studies attempting to evaluate a potential link between education and PD-MCI is discerning whether observed differences between education groups reflect different odds of cognitive decline or simply baseline differences in neuropsychological test performance. Longitudinal studies show that PD subjects with fewer YOE have greater cog- 
nitive decline over time [24], suggesting that differences in cognitive outcomes according to education level are not due to baseline testing differences alone. However, diagnosing PD$\mathrm{MCI}$ currently requires test scores to fall a certain standard deviation (usually $\geq 1.5 \mathrm{SD}$ ) below the adjusted mean, regardless of the baseline education level. This requires patients with higher baseline test scores to fall farther before reaching the impaired range. How this affects the diagnosis or interpretation of PD-MCI is unknown and cannot be determined from the current data.

Of our core neuropsychological measures, only the VVT z-scores, representing the executive function/cognitive flexibility domain, were significantly different between education levels. Although significant, the correlation between VVT scores and YOE was low (0.30). There was also a trend for higher JLO z-scores in the high-education group. In other studies investigating the effect of education on neuropsychological test scores in non-demented PD patients, subjects with higher education levels tended to perform better particularly in frontal/executive and visuospatial domains $[27,28]$, consistent with our findings. The implications of this for diagnosing MCI deserve further study. It remains unclear whether education protects against decline in executive function or whether baseline and non-progressive difficulties with executive tasks in low-education groups result in a higher proportion being labeled as MCI based on these tests. Longitudinal studies would be needed to answer this question.

In addition to broad challenges in diagnosing PD-MCI and interpreting neuropsychological results as discussed above, specific limitations to our study include the use of an available cohort for analysis rather than recruiting a representative cross-sectional population. While the study design cannot prove a protective effect of IQ, this is supported by the fact that premorbid IQ necessarily precedes the development of PD and PD-MCI. The WTAR is an estimate of premorbid IQ but is validated for this use [7]. The sample size may have limited the ability to detect significant interactions between variables. As discussed above, our results may only be generalized to similarly educated populations. No subject was excluded based on WTAR standard scores, though 1 subject refused to take the WTAR and withdrew consent. The range of WTAR standard scores of our subjects (80-126) is consistent with predicted WTAR scores for US adults with $\leq 8$ to $\geq 17$ YOE [29]. Thus, the WTAR inclusion criterion does not substantially limit the North American generalizability of the findings.

Given the broad CIs for ORs in the multivariable model, our study lacked the statistical precision to confirm or refute the protective effect of higher education suggested by univariate analysis and by the OR point estimates of education when considered as a dichotomous variable. In contrast, the beneficial association seen for IQ was robust. Education and estimated premorbid IQ were the only measured contributors to cognitive reserve. Data regarding type of employment and hobbies, other theorized contributors to cognitive reserve, were not collected and may have contributed further to our understanding of cognitive reserve and PD-MCI.

Our study found that higher education was associated with decreased odds of PD-MCI in univariate analysis, but not multivariable analysis. Estimated premorbid IQ was associated with substantially decreased odds of PD-MCI across all models. This finding is consistent with the cognitive reserve theory in the context of PD-MCI but suggests that IQ may have more influence than education level. Future studies should report estimated premorbid IQ in addition to education level and should also investigate whether other factors hypothesized to influence cognitive reserve, such as employment and/or hobbies, may also have protective roles, likely requiring a large sample size. Whether there is any protective benefit of IQ and other factors associated with cognitive reserve in delaying the progression from PD-MCI to PD dementia remains to be established. 


\section{Acknowledgements}

The study 'Validating the Montreal Cognitive Assessment for the diagnosis of Mild Cognitive Impairment in Parkinson's disease' is funded by The Michael J. Fox Foundation for Parkinson's Research. Melissa J. Armstrong received support as an Edmond J. Safra fellow at Toronto Western Hospital while working on this project. Dr. Naglie receives funding from the University of Toronto Mary Trimmer Chair in Geriatric Medicine and research support from the Canadian Institutes of Health Research (IAO-90399). Dr. Duff-Canning has research support from the Michael J. Fox Foundation, National Parkinson Foundation, and Canadian Institutes of Health Research. Dr. Eslinger has research support from the NIH (NS060722, ES019672, AG027771) and the Pennsylvania Department of Health. Dr. Zadikoff has received consultancy and honoraria from Teva, GSK, Merz, Allergan, Ipsen, and Abbott. She received research support from Parkinson Alliance. Dr. Mapstone has received honoraria from the Michael J. Fox Foundation, and funding from the NIH, Michael J. Fox Foundation, Neurologix, Inc., and Advanced Neuromodulation System, Inc. Dr. Chou receives research support from the NIH (NS44504-08), participates as a site-PI in clinical trials sponsored by the Huntington Study Group (PHAROS, 2CARE, and CREST-HD), receives royalties from UpToDate, and has received honoraria from Allergan for speaking engagements, and from Medtronic and Merz Pharmaceuticals for consulting work. Dr. Persad is a co-investigator on NIH grant P01 NS015655-26A1 and co-PI on MCASTL RFP2010-04. Dr. Litvan receives research support from the NIH (R01 PAS-03-092 NIA), National Parkinson Foundation, Parkinson Support Center of Kentuckiana, CurePSP, Noscira, Allon Therapeutics, and Parkinson Study Group. Dr. Fox has research support from the Parkinson Society Canada, Michael J. Fox Foundation, and NIH. She has served as a consultant for Teva, Novartis, Santhera, Merz, Merck Serono, and Bristol-Myers Squibb. Dr. Marras is a recipient of a New Investigator Award from the Canadian Institutes of Health Research. She also receives funding from the Michael J. Fox Foundation, National Parkinson Foundation, Merck Serono, and Allon Therapeutics. The authors would like to thank Dr. Nancy Kennedy and Dr. Paul J. Eslinger, who both served as local investigators, and Brandon Rothberg, who provided database and administrative assistance throughout the study.

\section{Disclosure Statement}

No author has conflicts of interest related to this paper.

\section{References}

1 Stern Y: Cognitive reserve. Neuropsychologia 2009;47:2015-2028.

-2 Caviness JN, Driver-Dunckley E, Connor DJ, Sabbagh MN, Hentz JG, Noble B, Evidente VG, Shill HA, Adler CH: Defining mild cognitive impairment in Parkinson's disease. Mov Disord 2007;22: 1272-1277.

-3 Mamikonyan E, Moberg PJ, Siderowf A, Duda JE, Have TT, Hurtig HI, Stern MB, Weintraub D: Mild cognitive impairment is common in Parkinson's disease patients with normal Mini-Mental State Examination (MMSE) scores. Parkinsonism Relat Disord 2009;15:226-231.

-4 Sollinger AB, Goldstein FC, Lah JJ, Levey AI, Factor SA: Mild cognitive impairment in Parkinson's disease: subtypes and motor characteristics. Parkinsonism Relat Disord 2010;16:177-180.

-5 McKinlay A, Grace RC, Dalrymple-Alford JC, Roger D: Cognitive characteristics associated with mild cognitive impairment in Parkinson's disease. Dement Geriatr Cogn Disord 2009;28:121-129. 
Armstrong et al.: Roles of Education and IQ in Cognitive Reserve in Parkinson's

Disease-Mild Cognitive Impairment

6 Wechsler D: Manual for the Wechsler Memory Scale, ed 3. New York, The Psychological Corporation, 1997.

7 Strauss E, Sherman EMS, Spreen O: A Compendium of Neuropsychological Tests: Administration, Norms, and Commentary, ed 3. New York, Oxford University Press, 2006.

8 Delis DC, Kaplan E, Kramer JH: Examiner's Guide for the Delis-Kaplan Executive Function System. San Antonio, The Psychological Corporation, 2001.

9 Benton AL, Sivan AB, Hamsher KD, Varney NR, Spreen O: Contributions to Neuropsychological Assessment, ed 2. Orlando, Psychological Assessment Resources, 1994.

10 Meyers JE, Meyers KR: Rey Complex Figure Test and Recognition Trial - Professional Manual. Odessa, Psychological Assessment Resources, 1995.

11 Delis DC, Kramer JH, Kaplan E, Ober BA: Manual for the California Verbal Learning Test, ed 2. San Antonio, The Psychological Corporation, 2002.

-12 Taylor AE, Saint-Cyr JA, Lang AE: Memory and learning in early Parkinson's disease: evidence for a 'frontal lobe syndrome'. Brain Cogn 1990;13:211-232.

$\checkmark 13$ Wicklund AH, Johnson N, Weintraub S: Preservation of reasoning in primary progressive aphasia: further differentiation from Alzheimer's disease and the behavioral presentation of frontotemporal dementia. J Clin Exp Neuropsychol 2004;26:347-355.

14 Grace J, Malloy PF: Frontal Systems Behavior Scale (FrSBe) Professional Manual. Lutz, Psychological Assessment Resource, 2001.

-15 Williams BW, Mack W, Henderson VW: Boston Naming Test in Alzheimer's disease. Neuropsychologia 1989;27:1073-1079.

-16 Babyak MA: What you see may not be what you get: a brief, nontechnical introduction to overfitting in regression-type models. Psychosom Med 2004;66:411-421.

-17 Peduzzi P, Concato J, Kemper E, Holford TR, Feinstein AR: A simulation study of the number of events per variable in logistic regression analysis. J Clin Epidemiol 1996;49:1373-1379.

- 18 Harrell FE Jr, Lee KL, Califf RM, Pryor DB, Rosati RA: Regression modelling strategies for improved prognostic prediction. Stat Med 1984;3:143-152.

-19 Nasreddine ZS, Phillips NA, Bédirian V, Charbonneau S, Whitehead V, Collin I, Cummings JL, Chertkow H: The Montreal Cognitive Assessment, MoCA: a brief screening tool for mild cognitive impairment. J Am Geriatr Soc 2005;53:695-699.

20 Akaike H: A new look at the statistical model identification. IEEE Trans Automat Control 1974;19: $716-723$.

- 21 Alexander GE, Furey ML, Grady CL, Pietrini P, Brady DR, Mentis MJ, Schapiro MB: Association of premorbid intellectual function with cerebral metabolism in Alzheimer's disease: implications for the cognitive reserve hypothesis. Am J Psychiatry 1997;154:165-172.

22 OECD: Education at a Glance 2011. OECD Publishing, 2011.

-23 Janvin C, Aarsland D, Larsen JP, Hugdahl K: Neuropsychological profile of patients with Parkinson's disease without dementia. Dement Geriatr Cogn Disord 2003;15:126-131.

-24 Muslimovic D, Schmand B, Speelman JD, de Haan RJ: Course of cognitive decline in Parkinson's disease: a meta-analysis. J Int Neuropsychol Soc 2007;13:920-932.

25 Pai MC, Chan SH: Education and cognitive decline in Parkinson's disease: a study of 102 patients. Acta Neurol Scand 2001;103:243-247.

- 26 Kummer A, Harsányi E, Dias FM, Cardoso F, Caramelli P, Teixeira AL: Depression impairs executive functioning in Parkinson disease patients with low educational level. Cogn Behav Neurol 2009; 22:167-172.

-27 Green J, McDonald WM, Vitek JL, Evatt M, Freeman A, Haber M, Bakay RA, Triche S, Sirockman B, DeLong MR: Cognitive impairments in advanced PD without dementia. Neurology 2002;59:1320 1324.

-28 Cohen OS, Vakil E, Tanne D, Nitsan Z, Schwartz R, Hassin-Baer S: Educational level as a modulator of cognitive performance and neuropsychyatric features in Parkinson disease. Cogn Behav Neurol 2007;20:68-72.

29 Wechsler Test of Adult Reading Manual. San Antonio, The Psychological Corporation, 2001. 